\title{
What survived from the PLANAFLORO Project: Angiosperms of Rondônia State, Brazil
}

\author{
Samuel Carleial ${ }^{1^{*}}$ and Narcísio C. Bigio ${ }^{2}$ \\ 1 University of Konstanz, Department of Biology, M842, PLZ 78457, Konstanz, Germany. \\ 2 Universidade Federal de Rondônia, Campus José Ribeiro Filho, BR 364, Km 9.5, CEP 76801-059. Porto Velho, RO, Brasil. \\ * Corresponding author. E-mail: samuel.carleial@gmail.com
}

\begin{abstract}
The Rondônia Natural Resources Management Project (PLANAFLORO) was a strategic program developed in partnership between the Brazilian Government and The World Bank in 1992, with the purpose of stimulating the sustainable development and protection of the Amazon in the state of Rondônia. More than a decade after the PLANAFORO program concluded, the aim of the present work is to recover and share the information from the long-abandoned plant collections made during the project's ecological-economic zoning phase. Most of the material analyzed was sterile, but the fertile voucher specimens recovered are listed here. The material examined represents 378 species in 234 genera and 76 families of angiosperms. Some 8 genera, 68 species, 3 subspecies and 1 variety are new records for Rondônia State. It is our intention that this information will stimulate future studies and contribute to a better understanding and more effective conservation of the plant diversity in the southwestern Amazon of Brazil.
\end{abstract}

\section{INTRODUCTION}

In early 1990, Brazilian Amazon was facing remarkably high rates of forest conversion (Laurance et al. 2001; INPE 2012). This was particularly true in the state of Rondônia where cattle ranching, poorly planned settlement, logging, and mining were threatening the local environments and dramatically changing the landscape (Pedlowski et al. 1997).

Following heavy criticism of the unsuccessful POLONOROESTE Project in the 1980s, the Brazilian Federal Government undertook a second effort to control biodiversity loss in Rondônia State. Therefore it conceived a plan in partnership with the World Bank to "implement an improved approach to natural resource management, conservation and development in the state": the Rondônia Natural Resources Management Project, also known as PLANAFLORO (World Bank 1992).

The project had an initial budget of US $\$ 167$ million and was implemented from 1993 to 2001 . It consisted of a series of strategic activities, focusing on land use, biodiversity conservation, farming systems, and agroecological and socioeconomic zoning (World Bank 2003). PLANAFLORO is appropriately credited with several important achievements, such as the creation of conservation units and the improvement of the local transportation infrastructure. Numerous criticisms, however, were still made by national and international experts, especially social NGOs, which had extremely limited participation in the project (World Bank 1995; Rodrigues 2002). For instance, critics highlighted the increase in deforestation during the implementation of PLANAFLORO (Browder et al. 2008) and thereafter. From 1990 to 2000, Rondônia State lost 26,247 $\mathrm{km}^{2}$ of forests, corresponding to $12.94 \%$ of its natural coverage. By 2011 , it was estimated that nearly $46 \%$ or almost half of the state's forests had been lost (Euler et al. 2008; INPE 2012).
The PLANAFLORO Project funded botanical expeditions in different areas of the state to inventory arboreal plants with potential use in the wood industry. The aim of the present study was to recover plant specimens and data collected during PLANAFLORO's ecological-economic zoning study between 1996 and 1997 and to disseminate these data and a preliminary checklist of the angiosperm trees in Rondônia State.

\section{Materials ANd Methods}

Unfortunately, the plant collections made during PLANAFLORO are dispersed. After almost 15 years neglected, less than half (870) of the originally 4000 vouchers were fertile and relatively undamaged. That material was decontaminated with a combination of $70 \%$ ethanol and $0.2 \%$ sodium hypochlorite. Then, it was frozen two times for three days, with a three-day interval, before transference to the main herbarium collection. Finally, specimens were registered, mounted, and deposited under an official voucher number at the Herbário Rondoniense João Geraldo Kuhlmann (RON) of the Federal University of Rondônia (UNIR). The vouchers are available digitally at the RON website (http://www.ron.unir.br).

To gather the information from the specimens, all available labels and identification lists were used. Collection sites, when specified, included protected areas, riparian zones, highways and farms, although we only mention here the entire municipality (Figure 1). Some specimens collected by Hamilton S. Pereira, however, lacked crucial information about site or date of collection and were only included to indicate new records for Rondônia State [see Table 1, tagged as (?)]. In addition, further information on date of collection, common name, and collector name and number were also recovered, when possible. The collector names were Araújo J., Eloísio Vinha, Dirlei (DRL), Marco Antonio, Martins, F. Almeida, L. Prestes and Hamilton S. Pereira. 


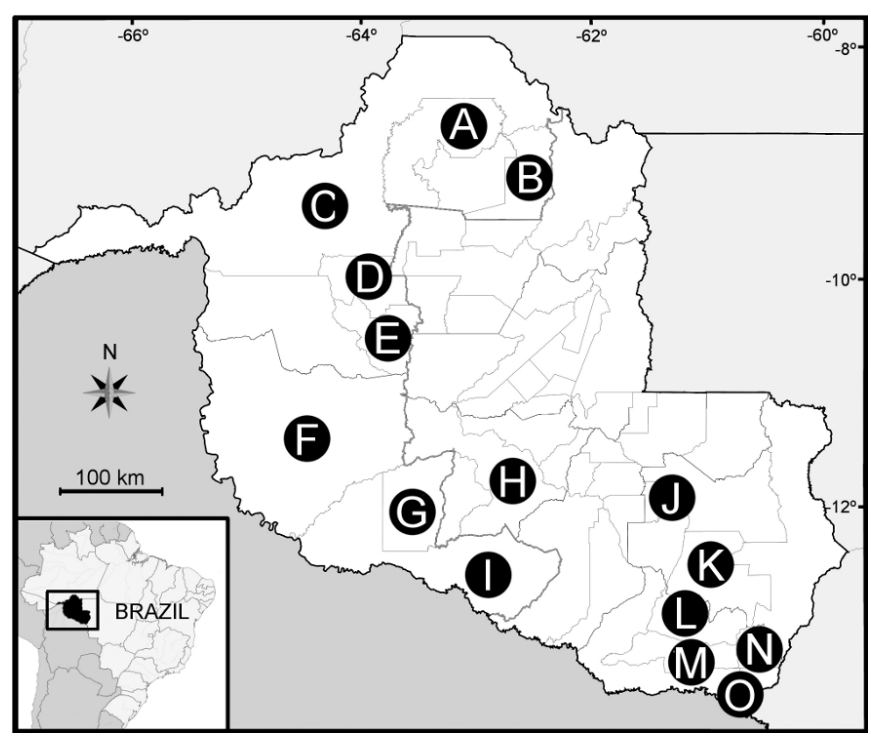

FIGURE 1. State of Rondônia, Brazil, showing the municipalities where field collections were carried out by PLANAFLORO during the 1990s. Sites were as follows: (a) Candeias do Jamari, (b) Cujubim, (c) Porto Velho, (d) Buritis, (e) Campo Novo de Rondônia, (f) Guajará Mirim, (g) São Francisco do Guaporé, (h) São Miguel do Guaporé, (i) Costa Marques, (j) Primavera de Rondônia, (k) Chupinguaia, (l) Corumbiara, (m) Cerejeiras, (n) Colorado do Oeste, and (o) Cabixi.

For the checklist, we have included only fertile vouchers, i.e. bearing flower or fruits (Figure 2A). Sterile material was deposited in a secondary collection (Figure 2B) under different voucher numbers [ex: RON e1721, Abarema jupunba (Willd.) Britton \& Killip], also available online.

Plant determination was carried out in 1997 by Nelson de Araújo Rosa, of the Museu Paraense Emílio Goeldi (MPEG). Burseraceae was revised by Douglas Daly (NYBG), while Peraceae was revised by Narcísio C. Bigio (UNIR). In addition, species names were double-checked against Tropicos (http://www.tropicos.org) and The Plant List 2010 (http://www.theplantlist.org) websites to avoid nomenclatural confusion. New records for the region were based on the online Lista de Espécies da Flora do Brasil 2012 (http://floradobrasil.jbrj.gov.br/) and the Herbário Virtual da Flora e dos Fungos (INCT- HVFF) (http://inct. splink.org.br) websites. Finally, voucher duplicates were sent to the CEN, IAN, INPA, MG, and RB herbaria (acronyms according to Holmgren et al. 1990).

\section{RESULTS AND Discussion}

We recorded 378 species distributed in 234 genera and 76 families of angiosperms (see Table 1). The most species-rich families found were Annonaceae (21 spp.), Leguminosae - Caesalpinioideae (21 spp.), and Moraceae (19 spp.), followed by Euphorbiaceae and Rubiaceae, each with $18 \mathrm{spp}$. (Figure 3). The remaining families, which made up $75 \%$ of the species, each presented 17 species or fewer, and ca. $42 \%$ of these were each represented by a single species. Casearia (Salicaceae) (9 spp.), Protium (Burseraceae), and Inga (Leguminosae - Mimosoideae) (8 spp.) were the richest genera, followed by Guarea (Meliaceae) and Miconia (Melastomataceae) each with 7 species (Figure 4).

Our results showed 72 new records for Rondônia State, including 8 genera, 68 species, 3 subspecies, and 1 variety. Approximately $20 \%$ of the specimens recovered from PLANAFLORO collections constituted new records, underscoring the lack of knowledge of the local Flora. For example, common species occurring in the state, like caranaí (Lepidocaryum tenue Mart.) or the fig tree Ficus maxima Mill. (pers. obs.) were not found in the database of the Brazilian Flora 2012 for Rondônia.

The PLANAFLORO Project aimed specifically to inventory arboreal species (World Bank 1992). Curiously, though, we found some characteristically herbaceous families, such as Commelinaceae and Costaceae. We can argue that these species were collected, because they were abundant inside the collection sites. Likewise, these species could have been perceived as economically important, regardless of their wood potential. The reason behind this, however, is still unclear.

To mention some of the herbs and shrubs collected, we found a Heliconia (Heliconia rostrata Ruiz \& Pav., Heliconiaceae), a cactus (Selenicereus sp., Cactaceae), and grasses (Lasiacis ligulata Hitchc. \& Chase and Olyra latifolia L., Poaceae). But, due to the bad conservation status and the lack of reproductive parts on these specimens which would allow an identification confirmation, we decided to exclude these records from our checklist.

Although records occurred in diverse municipalities, few species were found in more than one collection site. The most frequently collected species was Fusaea longifolia (Aubl.) Saff. (Annonaceae), found in six different sites. Comparisons with the Flora of the neighbor state of Acre, Brazil (Daly \& Silveira 2008), showed that 239 species occur on both states, representing over $60 \%$ of the species listed here [see Table 1 , tagged as $(\diamond)$ ].

In addition, some economically important trees have been observed in Rondônia but are not yet represented on the new checklist, including big-leaf mahogany (Swietenia macrophylla King) and cedar (Cedrela odorata L.). On the contrary, other common species were found, like jatobá (Hymenaea courbaril L., Leguminosae - Caesalpinioideae), Pará rubber [Hevea brasiliensis (Willd. ex A. Juss.) Müll. Arg., Euphorbiaceae], and Copaifera spp. (Leguminosae Caesalpinioideae), already reported as native species from Rondônia State by some surveys (Curi 2000; Bentes-Gama et al. 2008). This suggests that the material recovered from PLANAFLORO, although informative, could still be incomplete for the studied area.

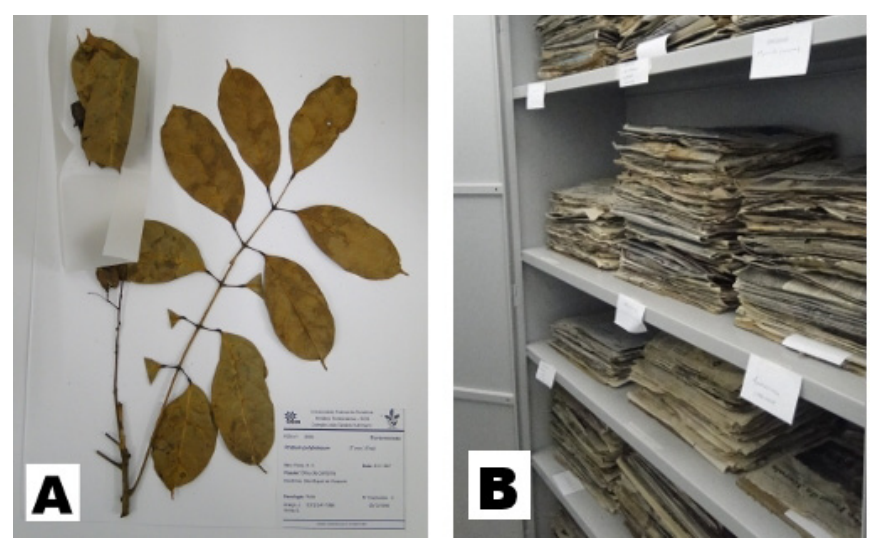

Figure 2. PLANAFLORO botanical collections deposited at the Herbário Rondoniense (RON). (A) Protium polybotryum (Turcz.) Engl., a new record for Rondônia and one of the few fertile vouchers found in reasonable conservation status. (B) The sterile collection is formed by more than 3000 vouchers. Specimens present only leaves and wood samples. 


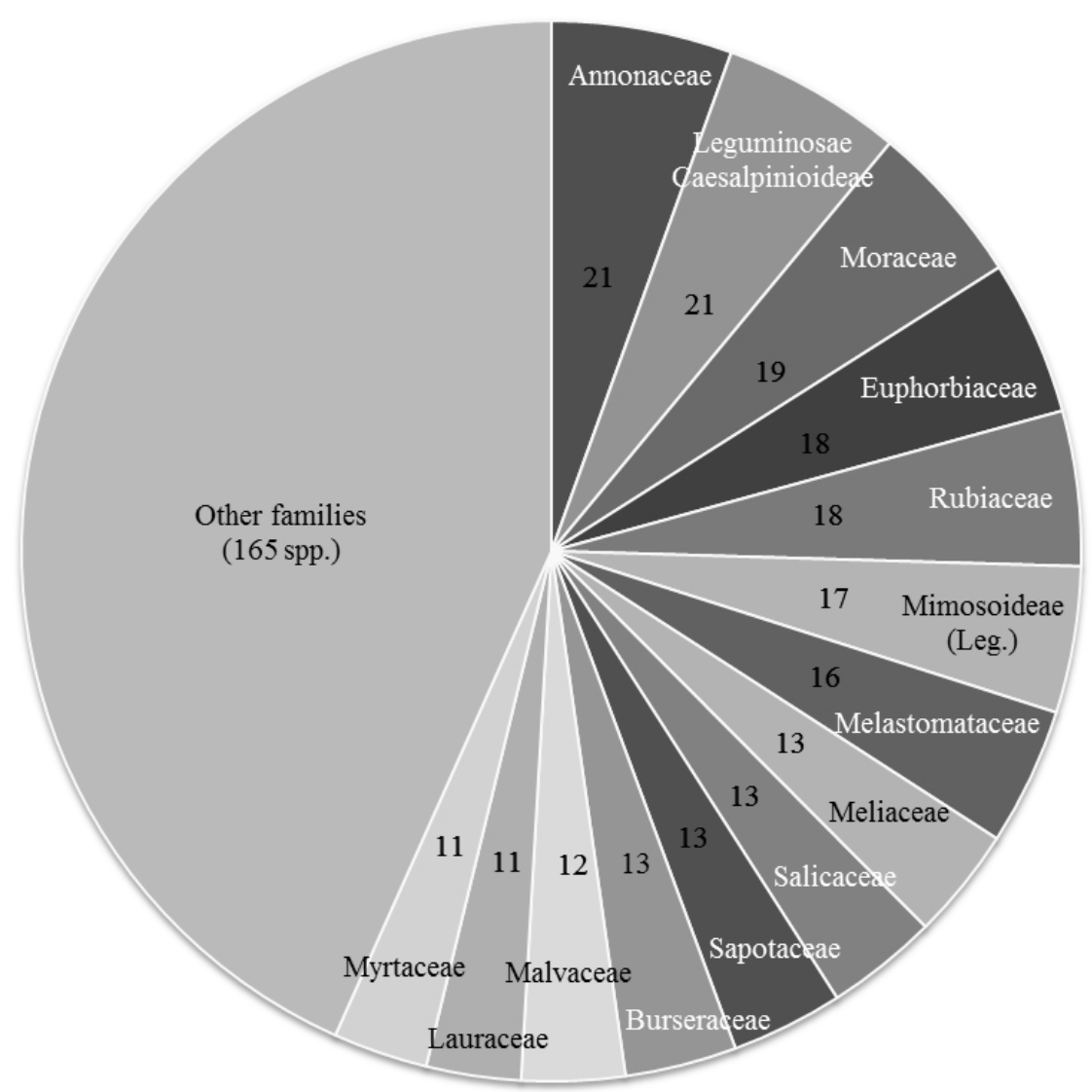

FIGURE 3. Most speciose families of angiosperms found in the PLANAFLORO collections. These presented from 21 (Annonaceae and Leguminosae Caesalpinioideae) to 11 (Myrtaceae and Lauraceae) species. The remaining 62 families corresponded to 82\% of total families and 166 species.

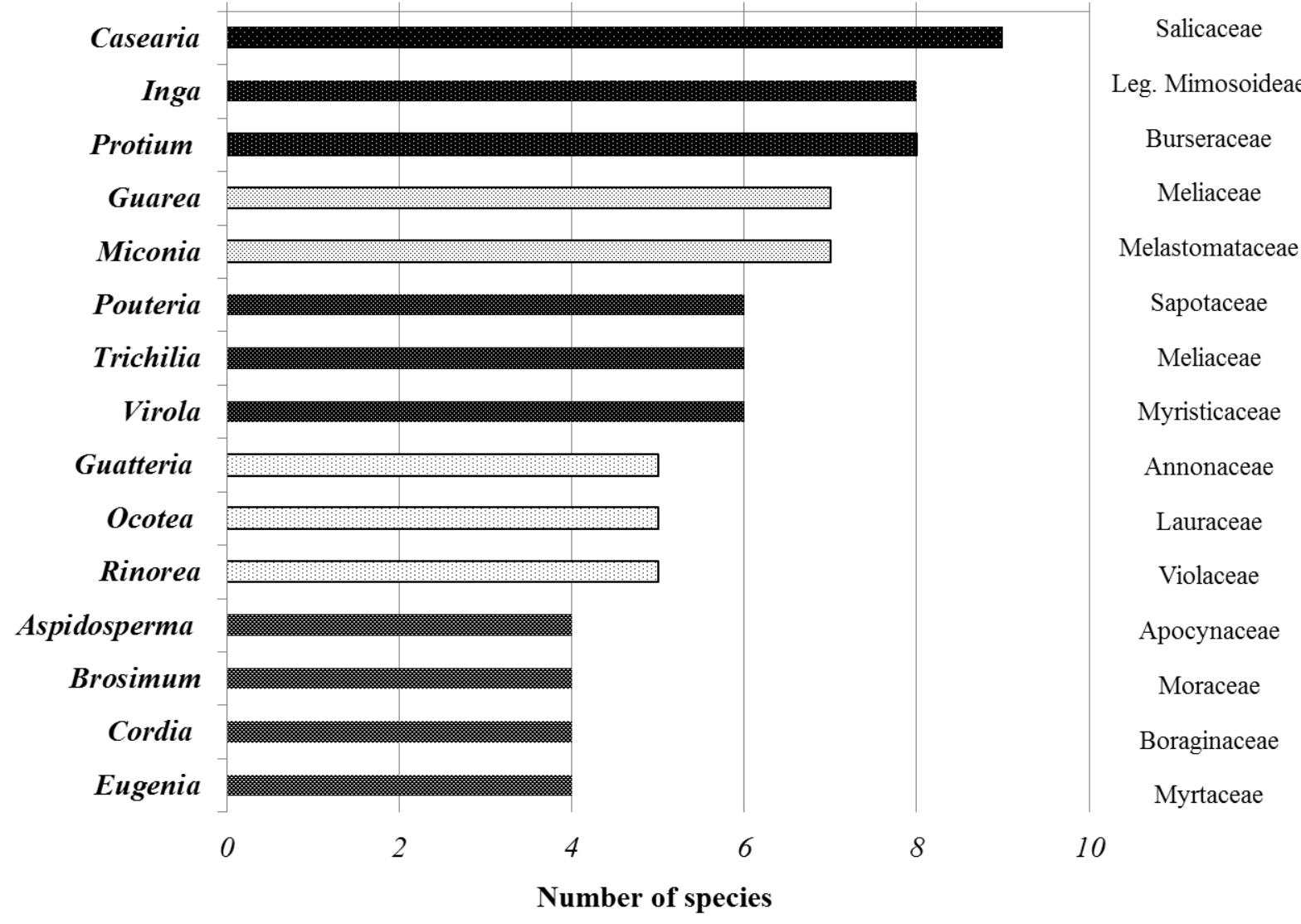

FIGURE 4. Richest genera found in the PLANAFLORO collections. Note that the most representative genera did not belong to the richest families. All genera presented less than 10 species and more than half of the 234 genera presented only one species. 
After the PLANAFLORO Project ended, local authorities produced a technical report on vegetation cover, as part of the ecological-economic zoning study (SEDAM 2007). The majority of the specimens were sent to the MG herbarium, while Poaceae and Asteraceae specimens were sent to the IBGE-DF and to the SPF herbarium, respectively. We have found specimens stored at the MG herbarium that were collected at the same sites in Rondônia State between 1996 and 1997. However, the field data for these have different collector names and dates different from those registered in our study. It is possible that these specimens belong to PLANAFLORO collections as well, but future surveys will be necessary to answer this question. Therefore, our list of plant species of Rondônia seems to be the first attempt to associate PLANAFLORO collections to a herbarium, making the plant information available publicly.

It is important to consider that forests of Rondônia have been under continuous human pressure over the past decades. While Pacaás Novos in Guajará Mirim (Figure 1F) is now officially conserved as a National Park, other forests in the state that were collection sites have been degraded or converted (Ribeiro et al. 2005). For instance, the zones of impact along the BR-319 and BR-421 highways, where Dirlei (DRL) and H. S. Pereira collected, have been seriously degraded by human activities (Euler et al. 2008). Nevertheless, since information about the local diversity in Rondônia is scarce (Brandon et al. 2005) and no effective survey has attended to the urgent need for a full inventory of local species, the data provided in this work is still of great value.

Remote and isolated areas, such as indigenous territories or agricultural frontiers, are usually overlooked by scientific research, and sadly, Rondônia State is not an exception. As daunting as the task might appear, however, the data from an intensive inventory of the botanical diversity of southwestern Brazilian Amazon can be used as an important tool to conserve its forests and plant diversity in the face of severe development pressures and unsustainable management of plant resources. We hope that this work will stimulate other flora-related studies in Rondônia in the near future, and perhaps it will constitute the first step toward a complete Flora of Rondônia.

TABLE 1. List of angiosperms from Rondônia State, Brazil. Some records are missing due to incomplete information on labels or determination lists. New records are discriminated in taxonomic level. (*): voucher number of the specimen deposited at the Herbário Rondoniense (RON). (?): voucher collected by Hamilton S. Pereira lacking information on site of collection. ( ): species which also occurs in the neighbor state of Acre, Brazil.

\begin{tabular}{|c|c|c|c|}
\hline SPECIES/FAMILY & AREA & *VOUCHER & NEW RECORD \\
\hline \multicolumn{4}{|l|}{ Achariaceae } \\
\hline Carpotroche longifolia (Poepp.) Benth. ( $\bullet$ & $\mathrm{e}$ & 3753 & \\
\hline Lindackeria latifolia Benth. & $\mathrm{e}$ & 3759 & \\
\hline Lindackeria paludosa (Benth.) Gilg (») & $\mathrm{e}$ & 4159 & \\
\hline \multicolumn{4}{|l|}{ Anacardiaceae } \\
\hline Spondias mombin L. & $\mathrm{c}$ & 3963 & \\
\hline \multicolumn{4}{|l|}{ Annonaceae } \\
\hline Anaxagorea phaeocarpa Mart. ( & $\mathrm{d}, \mathrm{n} / \mathrm{o}$ & 3723,3679 & \\
\hline Anaxagorea sp. & l & 3996 & \\
\hline Annona ambotay Aubl. ( $\bullet$ & $\mathrm{e}$ & 4137 & \\
\hline Annona montana Macfad. $(\diamond)$ & $\mathrm{d}$ & 4104 & \\
\hline Bocageopsis multiflora (Mart.) R.E. Fr. & $\mathrm{j}$ & 3776 & \\
\hline Duguetia flagellaris Huber & $\mathrm{d}$ & $3722 / 4090$ & \\
\hline Duguetia lepidota (Miq.) Pulle & $(?)$ & 3836 & species \\
\hline Ephedranthus sp. & $\mathrm{m}$ & 4037 & \\
\hline Fusaea longifolia (Aubl.) Saff. ( $\bullet$ & $c, d, f, h, j, m$ & $3890,4067,3952,2547,3787,4016$ & \\
\hline Guatteria discolor R.E. Fr. ( ) & $\mathrm{c}$ & 2632 & \\
\hline Guatteria poeppigiana Mart. & $\mathrm{e}$ & 3766 & species \\
\hline Guatteria recurvisepala R.E. Fr. ( $\bullet$ & $\mathrm{d}$ & 4077 & \\
\hline Guatteria schomburgkiana Mart. & $\mathrm{n} / \mathrm{o}$ & 3695 & \\
\hline Guatteria cf. olivacea R.E. Fr. ( ) & $d$ & 3719 & species \\
\hline Guatteria sp. & 1 & 3981 & \\
\hline Onychopetalum amazonicum R.E. Fr. & $\mathrm{c}$ & $2543 / 2599$ & \\
\hline Oxandra riedeliana R.E. Fr. ( $)$ & $\mathrm{c}, \mathrm{n} / \mathrm{o}$ & 3965,3701 & \\
\hline Oxandra xylopioides Diels ( $\bullet$ & $c, d, e$ & $2541,3712,4119$ & \\
\hline Xylopia amazonica R.E. Fr. & $\mathrm{d}$ & 4105 & \\
\hline Xylopia benthamii R.E. Fr. ( ) & $\mathrm{e}$ & 4133 & \\
\hline Xylopia cuspidata Diels ( & $\mathrm{d}$ & $3720 / 3721$ & \\
\hline Xylopia multiflora R.E.Fr. ( ) & $(?)$ & 3842 & \\
\hline \multicolumn{4}{|l|}{ Apocynaceae } \\
\hline Aspidosperma carapanauba Pichon & d & 4062 & \\
\hline Aspidosperma multiflorum A. DC. & $\mathrm{m}$ & 4026 & \\
\hline
\end{tabular}


TABle 1. Continued.

\begin{tabular}{|c|c|c|c|}
\hline SPECIES/FAMILY & AREA & *VOUCHER & NEW RECORD \\
\hline Aspidosperma nitidum Benth. ex Müll. Arg. & $\mathrm{c}$ & 2556 & species \\
\hline Aspidosperma rigidum Rusby ( $\bullet$ & $\mathrm{a} / \mathrm{b}$ & 2510 & \\
\hline Lacmellea floribunda (Poeppig.) Benth. & $\mathrm{c}$ & 2520 & species \\
\hline Macoubea guianensis Aubl. (») & $(?)$ & 3830 & \\
\hline Rauvolfia pentaphylla (Huber) Ducke & $\mathrm{n}$ & 3906 & \\
\hline Tabernaemontana angulata Mart. ex Müll. Arg. & $\mathrm{e}$ & 3760 & \\
\hline Tabernaemontana linkii A.DC. ( $\bullet$ & $\mathrm{f}$ & 2584 & \\
\hline Tabernaemontana undulata Vahl & $\mathrm{c}$ & 2528 & \\
\hline Tabernaemontana sp. & $\mathrm{h}$ & 2623 & \\
\hline \multicolumn{4}{|l|}{ Araceae } \\
\hline Heteropsis flexuosa var. flexuosa (Kunth) G.S.Bunting ( & $\mathrm{e}$ & 4122 & \\
\hline \multicolumn{4}{|l|}{ Araliaceae } \\
\hline Schefflera morototoni (Aubl.) Maguire, Steyerm. \& Frodin( $\bullet$ & $\mathrm{k}$ & 3803 & \\
\hline sp. 1 & $\mathrm{~h}$ & 2621 & \\
\hline sp. 2 & $\mathrm{~d}$ & 4047 & \\
\hline \multicolumn{4}{|l|}{ Arecaceae } \\
\hline Bactris sp. & 1 & 4010 & \\
\hline Geonoma sp. & 1 & $3997 / 4003$ & \\
\hline Lepidocaryum tenue var. gracile (Mart.) A.J.Hend. ( $\downarrow$ & $\mathrm{c}$ & 3962 & variety \\
\hline \multicolumn{4}{|l|}{ Asteraceae } \\
\hline Hebeclinium macrophyllum (L.) DC. (») & 1 & $3984 / 3990$ & \\
\hline \multicolumn{4}{|l|}{ Bignoniaceae } \\
\hline Handroanthus barbatus (E.Mey.) Mattos & c, e & 3877,4138 & \\
\hline Handroanthus heptaphyllus Mattos & i & 3931 & species \\
\hline Jacaranda copaia (Aubl.) D. Don ( & $\mathrm{a} / \mathrm{b}$ & 2515 & \\
\hline Jacaranda glabra (DC.) Bureau \& K.Schum. ( & $\mathrm{m}$ & 4019 & \\
\hline \multicolumn{4}{|l|}{ Bixaceae } \\
\hline Bixa arborea Huber & $(?)$ & 4187 & \\
\hline Cochlospermum orinocense (Kunth) Steud. ( $\bullet$ & $\mathrm{e}$ & 4163 & \\
\hline \multicolumn{4}{|l|}{ Boraginaceae } \\
\hline Cordia alliodora (Ruiz \& Pav.) Oken ( $\bullet$ & $\mathrm{a} / \mathrm{b}$ & 2517 & \\
\hline Cordia exaltata Lam. ( & $\mathrm{e}$ & 3752 & \\
\hline Cordia nodosa Lam. ( $\bullet$ & $\mathrm{d}, \mathrm{e}$ & 3733,4145 & \\
\hline Cordia tetrandra Aubl. ( $\bullet$ & $\mathrm{c}$ & 3956 & \\
\hline \multicolumn{4}{|l|}{ Burseraceae } \\
\hline Crepidospermum goudotianum (Tul.) Triana \& Planch. ( & d, n/o & 4070,3696 & \\
\hline Protium decandrum (Aubl.) Marchand & $\mathrm{c}$ & 3881 & \\
\hline Protium krukovii Swart ( $\bullet$ & $\mathrm{c}, \mathrm{n}$ & 3818,3895 & \\
\hline Protium polybotryum (Turcz.) Engl. & $\mathrm{h}$ & 2609 & \\
\hline Protium rhynchophyllum Rusby ( $\bullet$ & 1 & 3977 & species \\
\hline Protium robustum (Swart) D.M. Porter ( $\bullet$ & $\mathrm{c}$ & 2502 & \\
\hline Protium sagotianum Marchand ( $\bullet$ & $\mathrm{f}, \mathrm{k}$ & 3943,3798 & \\
\hline Protium tenuifolium (Engl.) Engl. & $\mathrm{d}, \mathrm{n} / \mathrm{o}$ & 3732,3700 & \\
\hline Protium trifoliolatum Engl. & $(?)$ & 4179 & \\
\hline Tetragastris altissima (Aubl.) Swart ( $\bullet$ & $\mathrm{a} / \mathrm{b}, \mathrm{d}$ & 2516,4061 & \\
\hline Tetragastris panamensis (Engl.) Kuntze ( & $\mathrm{f}$ & 3944 & \\
\hline Trattinnickia burserifolia Mart. ( ) & $(?)$ & 3834 & \\
\hline Trattinnickia glaziovii Swart ( $)$ & $\mathrm{a} / \mathrm{b}$ & 2511 & \\
\hline \multicolumn{4}{|l|}{ Calophyllaceae } \\
\hline Calophyllum brasiliense Cambess. ( & $\mathrm{j}$ & 3774 & genus \\
\hline Caraipa densifolia Mart. ( $\bullet$ & $\mathrm{k}$ & 3805 & \\
\hline \multicolumn{4}{|l|}{ Cannabaceae } \\
\hline Trema micrantha (L.) Blume ( $)$ & $\mathrm{m}$ & 4041 & \\
\hline Trema sp. & $\mathrm{i}, \mathrm{k}$ & 3935,1596 & \\
\hline \multicolumn{4}{|l|}{ Capparaceae } \\
\hline Capparis frondosa Jacq.. & $\mathrm{d}$ & 4085 & species \\
\hline \multicolumn{4}{|l|}{ Caricaceae } \\
\hline Jacaratia spinosa (Aubl.) A. DC. ( ) & $\mathrm{f}, \mathrm{m}$ & 2577,4030 & \\
\hline
\end{tabular}


TABLE 1. Continued.

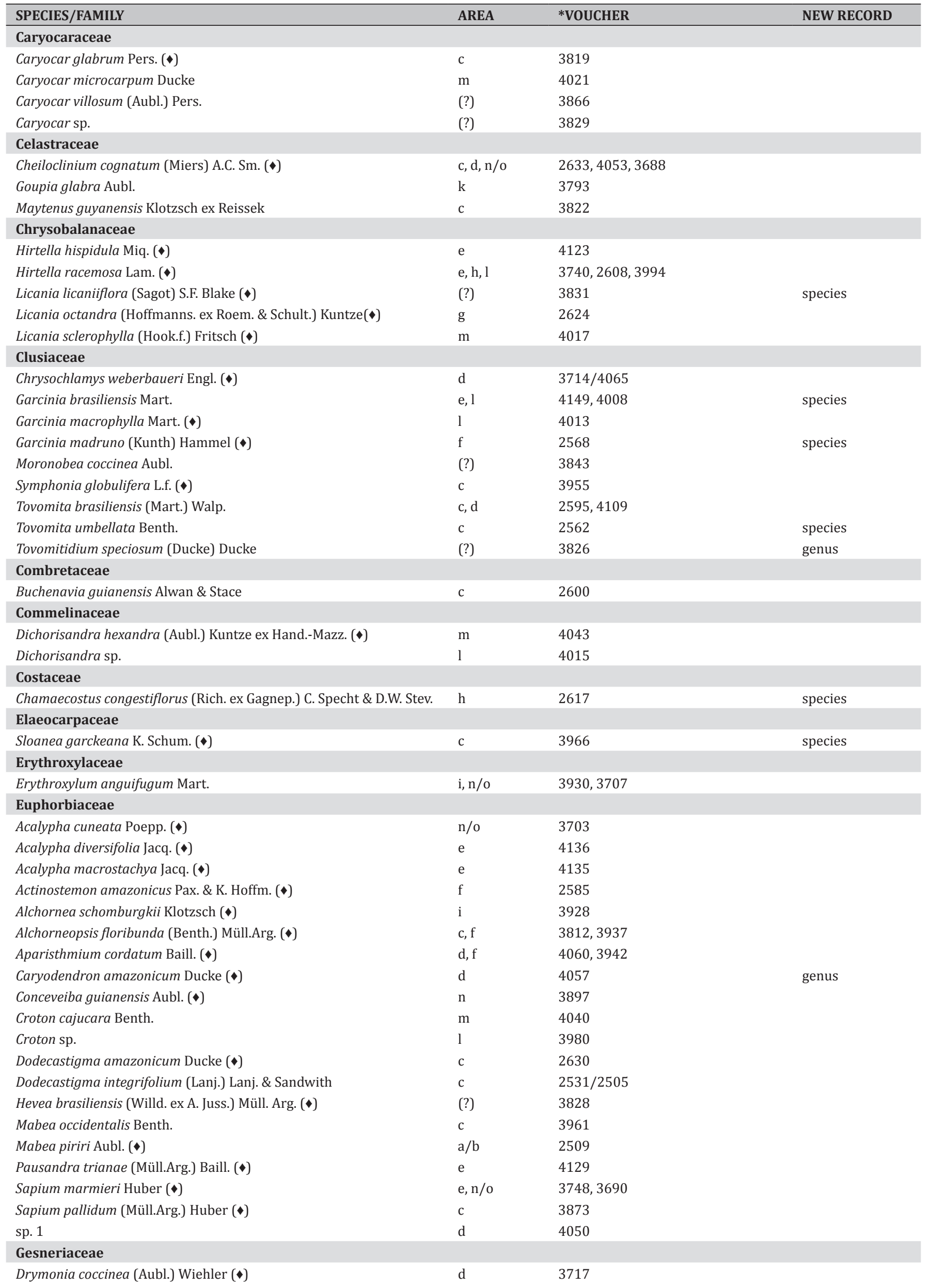


TABle 1. Continued.

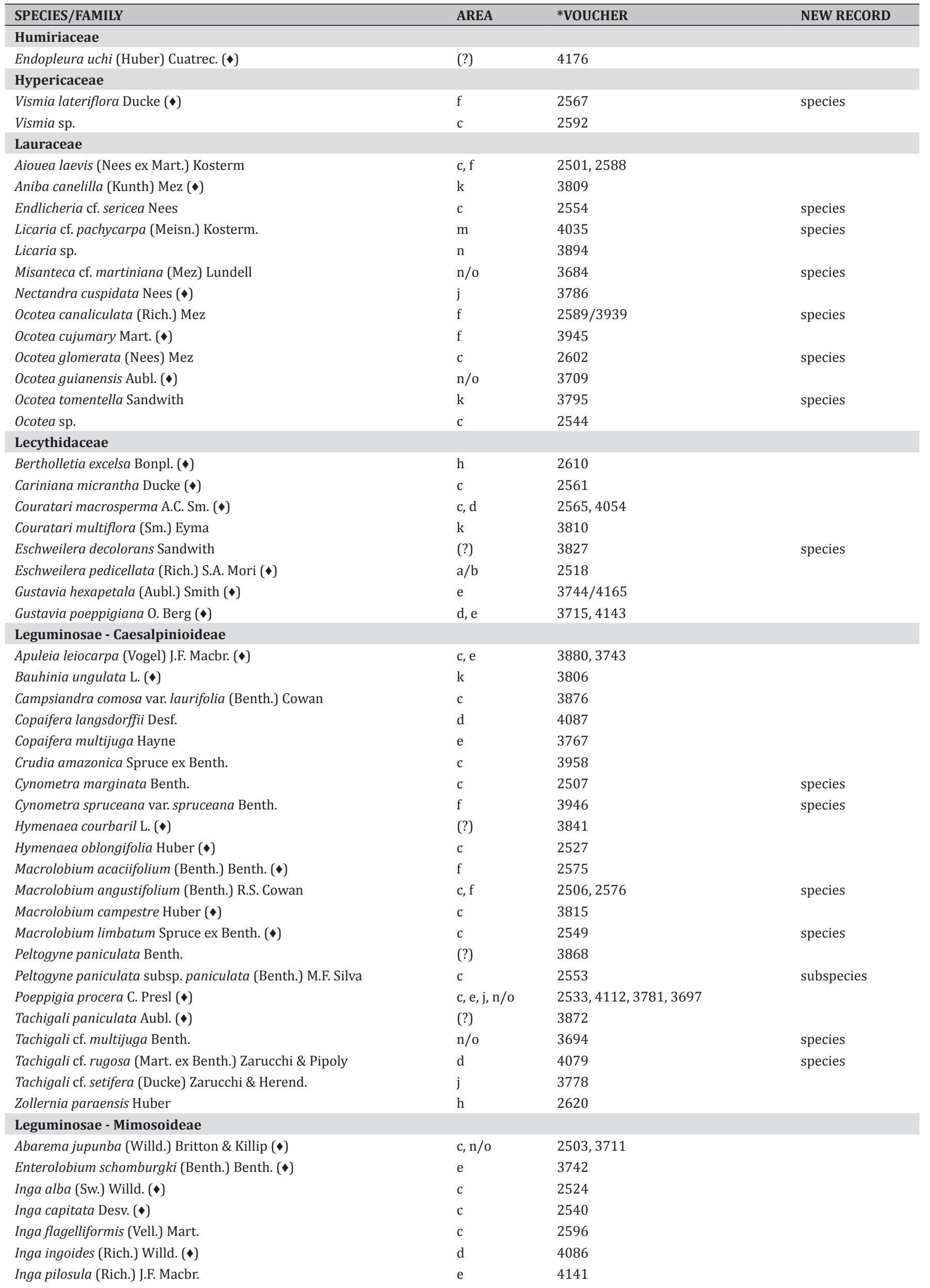


TABle 1. Continued.

\begin{tabular}{|c|c|c|c|}
\hline SPECIES/FAMILY & AREA & *VOUCHER & NEW RECORD \\
\hline Inga sertulifera DC. ( & $\mathrm{h}$ & 2615 & \\
\hline Inga thibaudiana DC. (») & $\mathrm{k}, \mathrm{m}$ & 3800,4031 & \\
\hline Inga cf. ruiziana G.Don ( $\bullet$ & $\mathrm{n} / \mathrm{o}$ & 3699 & \\
\hline Inga sp. & $\mathrm{d}, \mathrm{e}$ & 4076,4124 & \\
\hline Parkia multijuga Benth. (•) & $\mathrm{c}$ & 2521 & \\
\hline Parkia nitida Miq. ( & $\mathrm{c}$ & 2550 & \\
\hline Parkia cf. gigantocarpa Miq. & $\mathrm{c}$ & $2537 / 2545$ & species \\
\hline Pseudopiptadenia psilostachya (DC.) Brenan ( & $\mathrm{c}$ & 2522 & \\
\hline Senegalia sp. & $\mathrm{d}$ & 4084 & \\
\hline Stryphnodendron pulcherrimum (Willd.) Hochr. ( $\bullet$ & $\mathrm{c}$ & 2591 & \\
\hline Zygia inaequalis (Willd.) Pittier (•) & $\mathrm{d}$ & 3725 & \\
\hline sp. 1 & $\mathrm{n} / \mathrm{o}$ & 3686 & \\
\hline \multicolumn{4}{|l|}{ Leguminosae - Papilionoideae } \\
\hline Acosmium nitens (Vogel) Yakovlev & i & 3929 & \\
\hline Andira inermis (W. Wright) Kunth ex DC. ( $)$ & $\mathrm{i}$ & 3934 & \\
\hline Dinizia excelsa Ducke & $\mathrm{a} / \mathrm{b}$ & 2512 & \\
\hline Ormosia excelsa Benth. & $\mathrm{C}$ & $3816 / 3969$ & \\
\hline Ormosia paraensis Ducke & $\mathrm{m}$ & 4028 & \\
\hline Swartzia arborescens (Aubl.) Pittier ( & $\mathrm{h}$ & 2616 & \\
\hline Swartzia flaemingii var. psilonema (Harms) R.S. Cowan & $\mathrm{d}$ & 4096 & $\begin{array}{l}\text { species } \\
\text { variety }\end{array}$ \\
\hline Swartzia racemosa Benth. & $\mathrm{c}$ & 3967 & species \\
\hline Swartzia cf. laxiflora Bong. ex Benth. ( ) & $\mathrm{c}$ & 3813 & \\
\hline Taralea oppositifolia Aubl. ( $)$ & $\mathrm{c}$ & $3875 / 3964$ & \\
\hline \multicolumn{4}{|l|}{ Linaceae } \\
\hline Hebepetalum humiriifolium (Planch.) Benth. & $\mathrm{n}$ & 3905 & \\
\hline Roucheria columbiana Hallier f. & $\mathrm{c}$ & 3885 & species \\
\hline \multicolumn{4}{|l|}{ Loganiaceae } \\
\hline Potalia amara Aubl. ( $\bullet$ & $\mathrm{m}$ & 4027 & species \\
\hline \multicolumn{4}{|l|}{ Lythraceae } \\
\hline Physocalymma scaberrimum Pohl (») & $\mathrm{k}, \mathrm{n} / \mathrm{o}$ & 1591,3689 & \\
\hline \multicolumn{4}{|l|}{ Malpighiaceae } \\
\hline Byrsonima aerugo Sagot & $\mathrm{c}$ & 3886 & species \\
\hline Byrsonima altissima DC. & $\mathrm{m}$ & 4039 & species \\
\hline Byrsonima sp. & $\mathrm{i}, \mathrm{l}$ & 3936,3986 & \\
\hline sp. 1 & $\mathrm{f}$ & 3951 & \\
\hline \multicolumn{4}{|l|}{ Malvaceae } \\
\hline Apeiba albiflora Ducke & $\mathrm{d}, \mathrm{m}$ & 3734,4025 & \\
\hline Apeiba echinata Gaertn. & $c, f$ & 2504,3947 & \\
\hline Apeiba glabra Aubl. ( $\bullet$ & $\mathrm{e}$ & 4150 & species \\
\hline Ceiba crispiflora (Kunth) Ravenna & $\mathrm{a} / \mathrm{b}$ & 2514 & species \\
\hline Eriotheca globosa (Aubl.) A. Robyns ( & $\mathrm{k}$ & 3794 & \\
\hline Helicteres pentandra L. & $\mathrm{d}$ & 4080 & \\
\hline Heliocarpus americanus L. ( $\bullet$ & 1 & 4004 & \\
\hline Luehea speciosa Willd. & $\mathrm{e}$ & 4153 & species \\
\hline Matisia cordata Bonpl. ( $\bullet$ & $\mathrm{e}$ & 4147 & species \\
\hline Matisia ochrocalyx K. Schum. (») & $\mathrm{c}, \mathrm{e}$ & 2539,3756 & \\
\hline Matisia sp. & $\mathrm{n} / \mathrm{o}$ & 3698 & \\
\hline Mollia lepidota Spruce ex Benth. & $\mathrm{n} / \mathrm{o}$ & 3685 & \\
\hline Theobroma speciosum Willd. ex Spreng. ( $\bullet$ & $\mathrm{d}$ & 4095 & \\
\hline \multicolumn{4}{|l|}{ Marantaceae } \\
\hline Monotagma secundum (Petersen) Schum. & $\mathrm{d}$ & 3729 & species \\
\hline \multicolumn{4}{|l|}{ Marcgraviaceae } \\
\hline Norantea guianensis Aubl. & $\mathrm{h}$ & 2548 & \\
\hline \multicolumn{4}{|l|}{ Melastomataceae } \\
\hline Acinodendron burchellii (Triana) Kuntze & $\mathrm{n} / \mathrm{o}$ & 3705 & genus \\
\hline Bellucia aequiloba Pilg. ( & $\mathrm{c}$ & 2536 & species \\
\hline Graffenrieda rupestris Ducke ( $\bullet$ & $\mathrm{n}$ & 3901 & species \\
\hline Henriettella ovata Cogn. & $\mathrm{f}$ & 2579 & \\
\hline Loreya strigosa Gleason ( & $\mathrm{d}$ & 4069 & \\
\hline
\end{tabular}


TABle 1. Continued.

\begin{tabular}{|c|c|c|c|}
\hline SPECIES/FAMILY & AREA & *VOUCHER & NEW RECORD \\
\hline Meriania urceolata Triana & $\mathrm{c}$ & 2629 & \\
\hline Miconia affinis DC. ( $\bullet$ & $\mathrm{d}$ & 4078 & \\
\hline Miconia kappleri Naudin & $\mathrm{m}$ & 4190 & species \\
\hline Miconia minutiflora (Bonpl.) DC. & $(?)$ & 4173 & \\
\hline Miconia mirabilis (Aubl.) L.O. Williams & $\mathrm{n} / \mathrm{o}$ & 3706 & species \\
\hline Miconia poeppigii Triana ( $($ ) & $\mathrm{f}, \mathrm{k}$ & 2578,3799 & \\
\hline Miconia prasina (Sw.) DC. & $\mathrm{c}$ & 3821 & \\
\hline Miconia tomentosa (Rich.) D. Don ex DC. ( & $\mathrm{e}, \mathrm{m}$ & 4116,4189 & \\
\hline Miconia sp. & $\mathrm{c}$ & 2601 & \\
\hline Mouriri brachyanthera Ducke & $e, j$ & 3741,3788 & \\
\hline Mouriri collocarpa Ducke & $\mathrm{j}$ & 3790 & \\
\hline Mouririguianensis Aubl. & $e, j$ & 3769,3782 & \\
\hline \multicolumn{4}{|l|}{ Meliaceae } \\
\hline Guarea gomma Pulle (») & $\mathrm{k}$ & 3804 & \\
\hline Guarea grandifolia DC. ( $\bullet$ & $\mathrm{e}$ & 4118 & \\
\hline Guarea guidonia (L.) Sleumer ( $\bullet$ & $\mathrm{l}, \mathrm{m}$ & 3972,4038 & \\
\hline Guarea kunthiana A. Juss. ( & $\mathrm{d}, \mathrm{n}$ & 4089,3900 & \\
\hline Guarea pubescens (Rich.) A. Juss. ( & $\mathrm{d}, \mathrm{n} / \mathrm{o}$ & 4045,3687 & \\
\hline Guarea silvatica C.DC. ( ) & $\mathrm{d}$ & 4044 & \\
\hline Guarea velutina A.Juss. & $\mathrm{e}$ & 4142 & \\
\hline Trichilia cipo (A. Juss) C. DC. ( & $c, d$ & 2631,4088 & \\
\hline Trichilia martiana C. DC. & $\mathrm{h}$ & 2552 & \\
\hline Trichilia pleeana (A. Juss.) C. DC. ( ) & $\mathrm{k}, \mathrm{n} / \mathrm{o}$ & 1594,3683 & \\
\hline Trichilia quadrijuga Kunth ( & h, k & 2611,3801 & \\
\hline Trichilia septentrionalis C. DC. & $\mathrm{d}, \mathrm{l}$ & 4093,3971 & \\
\hline Trichilia cf. micrantha Benth. ( $)$ & d & 4049 & \\
\hline Trichilia sp. & d, e & 4099,4114 & \\
\hline \multicolumn{4}{|l|}{ Menispermaceae } \\
\hline Abuta grandifolia (Mart.) Sandwith ( $\bullet$ & $\mathrm{f}$ & 2573 & \\
\hline \multicolumn{4}{|l|}{ Monimiaceae } \\
\hline Mollinedia lanceolata Ruiz \& Pav. ( ) & 1 & 4000 & species \\
\hline \multicolumn{4}{|l|}{ Moraceae } \\
\hline Bagassa guianensis Aubl. & $\mathrm{l}, \mathrm{n} / \mathrm{o}$ & 4011,3704 & \\
\hline Brosimum acutifolium subsp. interjectum C.C. Berg & $\mathrm{f}$ & 3941 & $\begin{array}{l}\text { species and } \\
\text { subspecies }\end{array}$ \\
\hline Brosimum lactescens (S. Moore) C.C. Berg ( $\bullet$ ) & $(?)$ & 3835 & \\
\hline Brosimum potabile Ducke (») & $(?)$ & 3865 & \\
\hline Brosimum rubescens Taub. ( $\bullet$ & $\mathrm{c}$ & 3814 & \\
\hline Castilla ulei Warb. (») & $\mathrm{e}$ & 4157 & \\
\hline Clarisia ilicifolia (Spreng.) Lanj. \& Rossberg ( $\bullet$ ) & $\mathrm{m}$ & 4029 & \\
\hline Ficus insipida Willd. ( & $\mathrm{e}$ & 3751 & \\
\hline Ficus maxima Mill. (») & $\mathrm{n} / \mathrm{o}$ & 3691 & species \\
\hline Ficus paraensis (Miq.) Miq. (») & $\mathrm{h}$ & 2604 & \\
\hline Helicostylis pedunculata Benoist & d & 4064 & species \\
\hline Helicostylis tomentosa (Poepp. \& Endl.) Rusby ( $\bullet$ & j & 3772 & \\
\hline Naucleopsis caloneura (Huber) Ducke ( & $\mathrm{a} / \mathrm{b}, \mathrm{c}, \mathrm{e}, \mathrm{h}$ & $2513,2598,4158,2614$ & species \\
\hline Naucleopsis oblongifolia (Kuhlm.) Carauta ( & $\mathrm{c}$ & 3970 & species \\
\hline Perebea mollis subsp. mollis (Poepp. \& Endl.) Huber ( $\downarrow)$ & $\mathrm{c}$ & 2563 & \\
\hline Pseudolmedia laevigata Trécul ( $\bullet)$ & $c, d$ & 3959,3716 & \\
\hline Pseudolmedia laevis (Ruiz \& Pav.) J.F. Macbr. ( ) & $c, d, f$ & $2542,4075,3938$ & \\
\hline Sorocea guilleminiana Gaudich. (•) & $\mathrm{a} / \mathrm{b}$ & 2508 & \\
\hline Sorocea muriculata Miq. ( ) & $\mathrm{e}$ & 4160 & \\
\hline \multicolumn{4}{|l|}{ Myristicaceae } \\
\hline Campsoneura ulei Warb. ( $)$ & $d, f$ & 4082,2572 & \\
\hline Iryanthera juruensis Warb. (») & $c, d, e, j$ & $\begin{array}{l}2627,4081 \\
4131,3779\end{array}$ & \\
\hline Iryanthera macrophylla (Benth.) Warb. ( $\bullet$ & $(?)$ & 3833 & \\
\hline Osteophloeum platyspermum (Spruce ex A. DC.) Warb. ( & $(?)$ & $3847 / 3853$ & \\
\hline Virola calophylla (Spruce) Warb. $(\diamond)$ & $\mathrm{e}$ & 4130 & \\
\hline Virola elongata (Benth.) Warb. (») & $\mathrm{m}$ & 4024 & \\
\hline
\end{tabular}


TABle 1. Continued.

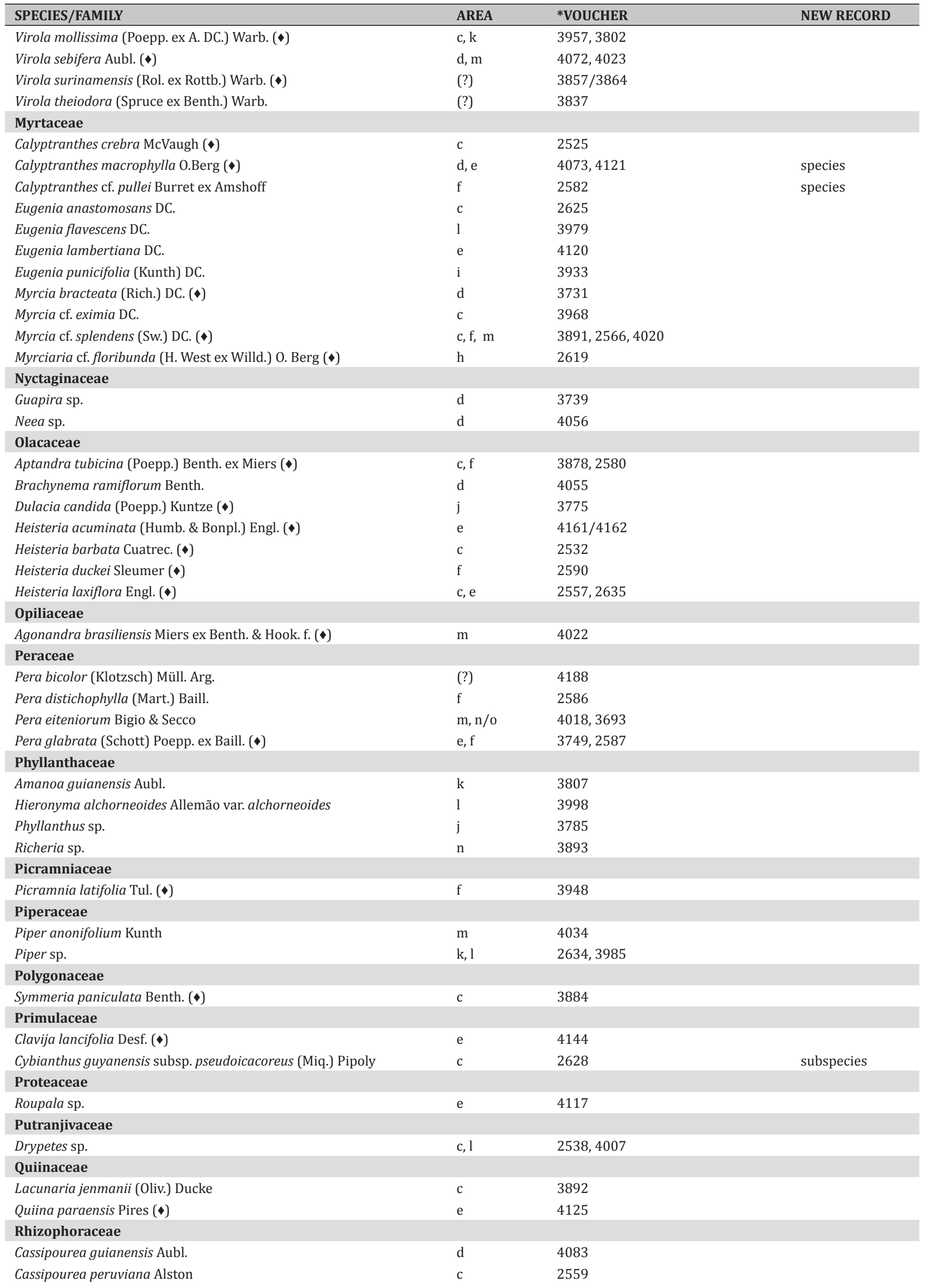


TABle 1. Continued.

\begin{tabular}{|c|c|c|c|}
\hline SPECIES/FAMILY & AREA & *VOUCHER & NEW RECORD \\
\hline Sterigmapetalum obovatum Kuhlm. & $\mathrm{j}$ & 3789 & \\
\hline \multicolumn{4}{|l|}{ Rubiaceae } \\
\hline Alibertia bertierifolia K. Schum. ( $\bullet$ & $\mathrm{f}$ & 2569 & \\
\hline Amaioua corymbosa Kunth ( $\bullet$ & $\mathrm{n} / \mathrm{o}$ & 3708 & \\
\hline Amaioua sp. & $\mathrm{c}, \mathrm{i}$ & 2597,3953 & \\
\hline Calycophyllum spruceanum (Benth.) Hook. f. ex K. Schum.( & $\mathrm{n} / \mathrm{o}$ & 3692 & species \\
\hline Capirona decorticans Spruce ( & $\mathrm{d}$ & 4059 & \\
\hline Carapichea ipecacuanha (Brot.) L.Andersson & $\mathrm{i}$ & 3932 & species \\
\hline Chassalia subspicata K. Schum. & $\mathrm{h}$ & 2607 & genus \\
\hline Cordiera macrophylla (K.Schum.) Kuntze & $\mathrm{l}, \mathrm{n}$ & 3992, 3899 & species \\
\hline Coussarea hirticalyx Standl. ( $\bullet$ & $(?)$ & 4178 & species \\
\hline Coussarea cf. macrophylla (Mart.) Müll.Arg. & $\mathrm{e}$ & 4140 & species \\
\hline Coussarea sp. & $\mathrm{c}$ & 2535 & \\
\hline Dialypetalanthus fuscescens Kuhlm. ( $)$ & $d, j, \quad l$ & $4052,3780,3982$ & \\
\hline Duroia sp. & h, l & 2606,4009 & \\
\hline Faramea glandulosa Poepp. \& Endl. ( $\bullet$ & $\mathrm{d}$ & 4101 & species \\
\hline Faramea sp. & $\mathrm{f}$ & 3949 & \\
\hline Genipa americana L. ( $\bullet$ & $\mathrm{c}$ & 2523 & \\
\hline Genipa sp. & 1 & 4012 & \\
\hline Hamelia sp. & 1 & 4001 & \\
\hline Isertia hypoleuca Benth. ( ) & $\mathrm{f}$ & 2571 & \\
\hline Psychotria poeppigiana Müll. Arg. ( $\downarrow)$ & $\mathrm{d}$ & 3724 & \\
\hline Psychotria cf. racemosa Rich. ( ) & $(?)$ & 4172 & \\
\hline Psychotria sp. & $\mathrm{d}, \mathrm{e}$ & 3713,4051 & \\
\hline Randia armata (Sw.) DC. ( $\bullet$ & $\mathrm{e}$ & 4139 & \\
\hline Rudgea sp. & $d$ & 4100 & \\
\hline Stachyarrhena acuminata Standl. ( $\downarrow)$ & $(?)$ & 4186 & \\
\hline Tocoyena cf. brasiliensis Mart. & $\mathrm{f}$ & 2570 & species \\
\hline \multicolumn{4}{|l|}{ Rutaceae } \\
\hline Conchocarpus ucayalinus (Huber) Kallunki \& Pirani ( $\bullet$ & $\mathrm{d}$ & 4058 & \\
\hline Dictyoloma vandellianum A.Juss. & $\mathrm{k}$ & 3808 & \\
\hline Galipea trifoliata Aubl. ( & $\mathrm{h}$ & 2622 & \\
\hline Galipea sp. & 1 & 3995 & \\
\hline Metrodorea flavida K. Krause ( $\bullet$ & c, d, i, l & $2626,4098,3927,3983$ & \\
\hline Zanthoxylum rhoifolium Lam. ( $\bullet$ & $\mathrm{m}$ & 4033 & \\
\hline Zanthoxylum cf. gardneri Engl. & $\mathrm{n} / \mathrm{o}$ & 3710 & species \\
\hline Zanthoxylum sp. & $\mathrm{e}, \mathrm{l}$ & 3764,3991 & \\
\hline \multicolumn{4}{|l|}{ Salicaceae } \\
\hline Casearia decandra Jacq. ( & $\mathrm{j}, \mathrm{n}$ & 3783,3896 & \\
\hline Casearia gossypiosperma Briq. ( $\bullet$ & $\mathrm{n}, \mathrm{n} / \mathrm{o}$ & 3898,3680 & \\
\hline Casearia grandiflora Cambess. & c & 2555 & \\
\hline Casearia guianensis (Aubl.) Urb. ( & $\mathrm{e}$ & 3746 & species \\
\hline Casearia javitensis Kunth $(\diamond)$ & $\mathrm{c}$ & 2564 & \\
\hline Casearia mariquitensis Kunth ( $\bullet$ & $\mathrm{d}$ & 3730 & \\
\hline Casearia negrensis Eichler & $\mathrm{d}$ & 4046 & \\
\hline Casearia pitumba Sleumer $(\bullet)$ & $\mathrm{h}$ & 2603 & \\
\hline Casearia ulmifolia Vahl ex Vent. & $\mathrm{j}, \mathrm{n} / \mathrm{o}$ & 3784,3681 & \\
\hline Hasseltia floribunda Kunth ( & $\mathrm{e}$ & $4155 / 4156$ & \\
\hline Laetia procera (Poepp.) Eichler ( & $(?)$ & 3832 & \\
\hline Pleuranthodendron lindenii (Turcz.) Sleumer $(\diamond)$ & d & 4094 & genus \\
\hline \multicolumn{4}{|l|}{ Sapindaceae } \\
\hline Allophylus floribundus (Poepp. \& Endl.) Radlk. ( $\bullet$ & $\mathrm{e}$ & $4152 / 4154$ & \\
\hline Cupania sp. & 1 & 3974 & \\
\hline Paullinia pinnata L. & 1 & 4005 & \\
\hline Talisia retusa R.S. Cowan ( & $\mathrm{c}$ & 3883 & \\
\hline \multicolumn{4}{|l|}{ Sapotaceae } \\
\hline Chrysophyllum argenteum subsp. auratum (Miq.) T.D. Penn.( () & $\mathrm{c}$ & 2546 & $\begin{array}{l}\text { species and } \\
\text { subspecies }\end{array}$ \\
\hline Chrysophyllum lucentifolium subsp. pachycarpum Pires \& T.D.Penn. ( ) & $(?)$ & 4166 & \\
\hline Chrysophyllum sp. & $\mathrm{n}$ & 3902 & \\
\hline
\end{tabular}


TABle 1. Continued.

\begin{tabular}{|c|c|c|c|}
\hline SPECIES/FAMILY & AREA & *VOUCHER & NEW RECORD \\
\hline Manilkara huberi (Ducke) Standl. & $\mathrm{c}$ & 2551 & \\
\hline Micropholis guyanensis (A.DC.) Pierre ( $\bullet$ & c & 3874 & \\
\hline Micropholis venulosa (Mart. \& Eichler) Pierre ( $\bullet$ & $\mathrm{k}$ & 3792 & \\
\hline Planchonella sp. & 1 & 4014 & genus \\
\hline Pouteria cladantha Sandwith ( $\bullet$ & $(?)$ & 3848 & \\
\hline Pouteria elegans (A. DC.) Baehni & $\mathrm{c}$ & 3728 & \\
\hline Pouteria gongrijpii Eyma & $(?)$ & 4174 & species \\
\hline Pouteria macrophylla (Lam.) Eyma ( $\bullet$ & $\mathrm{l}, \mathrm{m}$ & 4002,4036 & species \\
\hline Pouteria torta subsp. tuberculata (Sleumer) T.D. Penn. ( $\bullet$ & $\mathrm{c}$ & 2558 & subspecies \\
\hline Sarcaulus brasiliensis (A.DC.) Eyma ( & $(?)$ & 4182 & genus \\
\hline \multicolumn{4}{|l|}{ Simaroubaceae } \\
\hline Simaba orinocensis Kunth ( ) & c & 3882 & \\
\hline \multicolumn{4}{|l|}{ Siparunaceae } \\
\hline Siparuna cervicornis Perkins ( $(\bullet)$ & $\mathrm{h}$ & 2612 & species \\
\hline Siparuna decipiens (Tul.) A. DC. ( $\bullet$ & $e, f$ & 3765,2574 & \\
\hline Siparuna guianensis Aubl. ( $\bullet$ & $c, f$ & 3888,2583 & \\
\hline Siparuna thecaphora (Poepp. \& Endl.) A. DC. ( ) & $\mathrm{e}$ & 4115 & \\
\hline Siparuna sp. & $\mathrm{e}$ & 4113 & \\
\hline \multicolumn{4}{|l|}{ Solanaceae } \\
\hline Brunfelsia sp. & 1 & 3988 & \\
\hline Lycianthes sancti-caroli (H. Winkl.) Bitter & l & 3989 & species \\
\hline Solanum endopogon (Bitter) Bohs ( & $d$ & 3736 & \\
\hline Solanum cf. leucocarpon Dunal ( $\bullet$ & $\mathrm{m}$ & 4032 & \\
\hline Solanum sp. & 1 & 3987 & \\
\hline \multicolumn{4}{|l|}{ Staphyleaceae } \\
\hline Turpinia occidentalis (Sw.) G.Don ( & $c, d, e, l, n$ & $2519,4063,3755,3993,3903$ & \\
\hline \multicolumn{4}{|l|}{ Ulmaceae } \\
\hline Ampelocera edentula Kuhlm. ( ) & $\mathrm{e}$ & 4164 & \\
\hline \multicolumn{4}{|l|}{ Urticaceae } \\
\hline Pourouma bicolor subsp. bicolor Mart. ( & $\mathrm{f}$ & 3940 & \\
\hline Pourouma guianensis Aubl. ( $\bullet$ & $\mathrm{n}$ & 3904 & \\
\hline Pourouma velutina Mart. ex Miq. & $\mathrm{k}$ & $3796 / 3797$ & \\
\hline Pourouma cf. minor Benoist ( $\bullet$ & $\mathrm{e}$ & 4151 & \\
\hline Urera caracasana (Jacq.) Gaudich. ex Griseb. ( ) & $\mathrm{e}$ & 4148 & \\
\hline Urera laciniata Wedd. ( $\bullet$ & $\mathrm{d}$ & 3718 & species \\
\hline \multicolumn{4}{|l|}{ Violaceae } \\
\hline Leonia cymosa Mart. (») & $\mathrm{d}, \mathrm{e}$ & 4111,3754 & \\
\hline Leonia glycycarpa Ruiz \& Pav. ( ) & $c, j$ & 2593,3777 & \\
\hline Rinorea flavescens (Aubl.) Kuntze ( & $\mathrm{C}$ & 2560 & \\
\hline Rinorea lindeniana (Tul.) Kuntze ( & $\mathrm{e}$ & $4126 / 4127$ & \\
\hline Rinorea ovalifolia (Britton) S.F. Blake ( $\bullet$ & $\mathrm{d}$ & 3737 & \\
\hline Rinorea pubiflora (Benth.) Sprague \& Sandwith ( $\bullet$ & $\mathrm{h}$ & $2613 / 2618$ & \\
\hline Rinorea racemosa (Mart.) Kuntze ( & $(?)$ & 4168 & \\
\hline Rinoreocarpus ulei (Melch.) Ducke (») & $\mathrm{e}$ & 3761 & \\
\hline \multicolumn{4}{|l|}{ Vochysiaceae } \\
\hline Qualea acuminata Spruce ex Warm. ( $\bullet$ & $\mathrm{c}$ & 2526 & \\
\hline Qualea dinizii Ducke & $(?)$ & 3851 & \\
\hline Qualea grandiflora Mart. ( $\bullet$ & $\mathrm{n} / \mathrm{o}$ & 3682 & \\
\hline Vochysia surinamensis Stafleu & $(?)$ & 3870 & \\
\hline Vochysia vismiifolia Spruce ex Warm. & c & 3820 & \\
\hline \multicolumn{4}{|l|}{ Zingiberaceae } \\
\hline Renealmia monosperma Miq. ( $\bullet$ & $\mathrm{h}$ & 2605 & species \\
\hline
\end{tabular}


ACKNOWLEDGMENTS: This work was supported by the Conselho Nacional de Desenvolvimento Científico e Tecnológico (CNPq) and is part of the "REFLORA/CNPq Project: Integração, qualificação e disponibilização dos dados relacionados com coletas botânicas na Amazônia brasileira" coordinated by Michael J. G. Hopkins. In addition, this work would not be possible without the help of Antônio Laffayete da Silveira, who held the collection during the past 10 years. Finally, we would also like to thank the staff of the Herbário Rondoniense (RON) and the kind support of the Federal University of Acre-New York Botanical Garden collaborative research program, which sent a work party to RON funded by the JRS Biodiversity Foundation.

\section{LITERATURE CITED}

Bentes-Gama, M.M., N.S. Pereira, P.H.S. Capelasso, A.K.D. Salman and A.H. Vieira. 2008. Espécies arbóreas nativas com potencial para recuperação de áreas alteradas em Rondônia. Porto Velho: Embrapa Rondônia. 29 pp.

Brandon, K., G.A.B. Fonseca, A.B. Rylands and J.M.C. Silva. 2005. Conservação brasileira: desafios e oportunidades. Megadiversidade 1(1): 7-13.

Browder, J.O., M.A. Pedlowski and R. Walker. 2008. Revisiting Theories of Frontier Expansion in the Brazilian Amazon: A Survey of the Colonist Farming Population in Rondônia's Post-frontier, 1992-2002. World Development 36(8): 1469-1492.

Centro de Referência em Informação Ambiental, CRIA. 2012. Accessible at http://www.cria.org.br/. Captured on 20 July 2013.

Curi, W.J. 2000. Fortalecimento do setor madeireiro. Porto Velho: Federação das Indústrias do Estado de Rondônia, FIERO/SEBRAE. $164 \mathrm{pp}$.

Daly, D.C. and M. Silveira. 2008. First Catalogue of the Flora of Acre, Brazil/ Primeiro Catálogo da Flora do Acre, Brasil. Rio Branco: PRINTAC/ EDUFAC. $421 \mathrm{pp}$

Euler, A., B. Millikan, E.B. Brito, I.B. Cardozo, J.P. Leroy, L. Caminha, M.I. Hargreaves, R.V. Motta, S. Cunha, S. Matias, V. Alves and V. Glass. 2008. O Fim Da Floresta? - A Devastação das Unidades de Conservação e Terras Indígenas no Estado de Rondônia. Rondônia: Grupo de Trabalho Amazônico, GTA Rondônia. 62 pp.

Holmgren, P.K., N.H. Holmgren and L.C. Barnett. 1990. Index herbariorum. Part I: The herbaria of the world. $8^{\circ}$ Ed. New York: New York Botanical Garden. 693 pp.

INPE. 2012. Projeto Prodes - Monitoramento da Floresta Amazônica Brasileira por Satélite: Taxas anuais do desmatamento 1988 até 2011. Accessible at http://www.obt.inpe.br/prodes/. Captured on 25 February 2012.
Laurance, W.F., A.K.M. Albernaz and C. Costa. 2001. Is deforestation accelerating in the Brazilian Amazon? Environmental Conservation 28(4): 305-311

Lista de Espécies da Flora do Brasil. 2012. Accessible at http:// floradobrasil.jbrj.gov.br/2012. Captured on 8 February 2013.

Pedlowski, M.A., V.H. Dale, E.A.T. Matricardi and E.P. S. Filho. 1997. Patterns and impacts of deforestation in Rondônia, Brazil. Landscape and Urban Planning 38: 149-157.

Ribeiro, B., A. Veríssimo and K. Pereira. 2005. O Avanço do Desmatamento sobre as Áreas Protegidas em Rondônia. O Estado da Amazônia 6: $1-4$.

Rodrigues, M.G.M. 2002. Redes Transnacionais de Advocacia Pública: Estratégias e Impactos - 0 Projeto Planafloro e o Painel de Inspeção do Banco Mundial. Contexto Internacional 24(1): 73-128.

SEDAM. 2007. Zoneamento Socioeconômico-Ecológico do Estado de Rondônia: Um Instrumento de Gestão Ambiental a Serviço do Desenvolvimento Sustentável de Rondônia. Porto Velho: Secretaria de Estado do Desenvolvimento Ambiental do Governo do Estado de Rondônia. 323 pp.

The Plant List. 2010. Version 1. Published on the Internet. Accessible at http://www.theplantlist.org. Captured on 12 October 2012.

Tropicos.org 2012. Missouri Botanical Garden. Accessible at http://www. tropicos.org. Captured on 09 October 2012

World Bank. 1992. Brazil - Rondonia Natural Resource Management Project. Washington DC: World Bank. 94 pp.

World Bank. 1995. Request for Inspection of Rondonia Natural Resources Management Project (Loan No. 3444-BR) - Additional Review by the Inspection Panel. Washington DC: World Bank. 126 pp.

World Bank. 2003. Implementation Completion Report (CPL-34440) on a Loan in the Amount of US\$167.0 Million to the Federative Republic of Brazil for a Rondonia Natural Resources Management Project (Loan 3444-BR). Washington DC: World Bank. 69 pp.

RECEIVED: February 2013

ACCEPTED: December 2013

Published ONLINE: February 2014

EDITORIAL RESPONSIBILITY: Angelo Gilberto Manzatto 